\title{
Apple Picker: Computer software for studying human responding on concurrent and multiple schedules
}

\author{
WAYNE D. NORMAN and JERRY L. JONGERIUS \\ Northwestern College, Orange City, Iowa
}

\begin{abstract}
Apple Picker, a software package for the Apple II microcomputer, is described. Subjects respond by searching for "apples" in two "orchards." "Attempted pickings" may be reinforced according to random ratio or interval schedules. "Search" responses are recorded and may be examined either as rates of responding in the two orchards or visually as paths the subject has traced during the session.
\end{abstract}

Apple Picker and its associated programs allow one to investigate multiple and concurrent schedule responding. Human subjects respond on an Apple II+ or Apple IIe microcomputer. The multiple and concurrent schedules may consist of either random interval schedules of reinforcement or random ratio schedules of reinforcement, but not both simultaneously. In addition, there may be no more than two component schedules in any given experimental arrangement.

During a test session, subjects are presented with two screens ("orchards"), with only one orchard present at any one time. For multiple schedules, the computer controls when and for how long each orchard will be available. During concurrent schedules, the subject controls when and for how long each orchard will be available. Each orchard is composed of a grid of symbols ("trees"). The subject moves the screen cursor within the orchard from tree to tree. When the cursor is directly over a tree, that tree may be "picked" for an "apple." An apple will be found if the random ratio or random interval schedule requirements have been met for that orchard. The total number of apples found in each orchard is displayed at the bottom of the screen. If an apple is not found, then it is possible that a "worm" will be found. Worms eat a specified number of apples from the total number collected up to that point in that orchard. The probability of finding a worm may be set at a value ranging from 0.0 to 1.0. This value will then be in effect for both orchards for the entire test session. ${ }^{1}$

Apple Picker records all individual "search" moves through each orchard, a search move being defined as any movement of the cursor up, down, left, or right. In addition, Apple Picker records the number of attempted pickings, the number of apples found, the number of worms encountered, and the time spent in each orchard. These data are available in summary form for each orchard over

The authors' mailing address is: Department of Psychology, Northwestern College, Orange City, IA 51041. the entire test session and for each component presentation throughout the session.

As described below, Apple Picker has considerable flexibility as a research tool. Parameters may be varied to produce a large variety of experimental arrangements. In addition, all individual search moves and attempted pickings are recorded, allowing for considerable flexibility in data analysis. Within this context, Apple Picker was designed to investigate two phenomena: multiple schedule behavioral contrast and concurrent schedule matching. Multiple schedule behavioral contrast may be defined as the inverse relation between rate of responding in one component of a multiple schedule and the conditions of reinforcement in the other component (McSweeney, 1978). Concurrent schedule matching occurs when the relative rate of responding generated by each component of a concurrent schedule equals the relative rate of reinforcement that the component provides (Herrnstein, 1970; Norman \& McSweeney, 1978). Both phenomena have been extensively investigated with rats and pigeons as experimental subjects. Human subjects have been employed, typically in experimental situations in which the operant response is a buttonpress or lever pull. Often this type of experimental setting engenders low experimental realism and consequently low internal validity. Apple Picker was designed as an experimental paradigm that would yield higher experimental realism while maintaining high experimental control.

A few pilot studies have been conducted using Apple Picker. These studies have investigated such factors as instructions to subjects, range of parameter settings, session length, variability of subject responding, and subjects' reactions to the test situation. Investigation of multiple schedule behavioral contrast has just begun.

\section{SYSTEM REQUIREMENTS}

Apple Picker has been designed to run on the Apple II+, IIe line of computers. A Thunderclock clockcard must be installed in one of the six slots (2-7) of your 
Apple; Apple Picker automatically determines the slot in which it has been installed. A joystick is NOT required (in which case Apple Picker must be modified as described below), but it may be of help in some situations. ${ }^{2}$ For generating reports, a printer is not required, but is recommended. If a printer is used, it must be configured in slot one. The programs on the Apple Picker diskette are written in Applesoft BASIC and 6502 machine language. They require the DOS 3.3 operating system.

\section{OVERVIEW OF THE APPLE PICKER DISKETTE}

A catalog listing of the Apple Picker diskette is arranged as in Figure 1. It has been divided into five main sections.

\section{Major Programs}

All of the major programs needed to run Apple Picker are contained in this section of the catalog. It is not for data storage, other sections having been set aside for that purpose. Seven of the nine programs in this section are written in Applesoft BASIC; the other two are machine language programs that help Apple Picker run more efficiently.

The programs in this section include:

HELLO. This program runs automatically every time the diskette is placed in the drive and the Apple is turned on. It presents the experimenter with a menu of options such as the following:

$\begin{array}{ll} & \\ & \\ \langle 1\rangle & \text { APPLE PICKER } \\ \langle 2\rangle & \text { KEYBOARD RE-DEFINER } \\ \langle 3\rangle & \text { SYSTEM CONEIGURATION } \\ \langle 4\rangle & \text { REPORT GENERATOR } \\ \langle 5\rangle & \text { SYS POINTER } \\ \langle 6\rangle & \text { TRACKER }\end{array}$

RUN WHICH OF THESE PROGRAMS $(1-6)$ :

All that is necessary to run a program is to press the number of the chosen option. It is not necessary to press the return key.

APPLE PICKER. This program presents orchards, allows the subject to move the cursor and to attempt to pick apples, schedules the availability of apples and worms, and creates the data files to be used for later analysis of the subject's responding.

KEYBOARD REDEFINER. With this program, the experimenter may define the keys necessary to control Apple Picker, that is, the keys necessary to (1) move the cursor up, down, left, and right, (2) attempt to pick an apple, and, (3) change from one orchard to the other. The keys do not have to be the same for both the Apple IIe and Apple II +. Upon running this program, the experimenter is asked:
Re-define keys for which computer:

(1) Apple IIe

(2) Apple II +

Only the number of the chosen option should be pressed. After the data for the computer selected are read, the current defaults are presented. Changes may be made by answering the question "Any changes $(\mathrm{Y} / \mathrm{N})$ " with a "Y." Enter the new default values for the six options. To make a change, press a new letter; to accept the current (old) value, just press "return." When the six options have been entered, the program asks if the information is correct. If not, type " $N$ " to reenter the information.

SYSTEM CONFIGURATION. This program allows creation or modification of an existing System Data File (described below). These files specify the experimental parameters for Apple Picker, such as the percentage of apples found in orchards $A$ and $B$ and whether an interval or ratio schedule will be employed. A sample System Data File is presented in Figure 2.

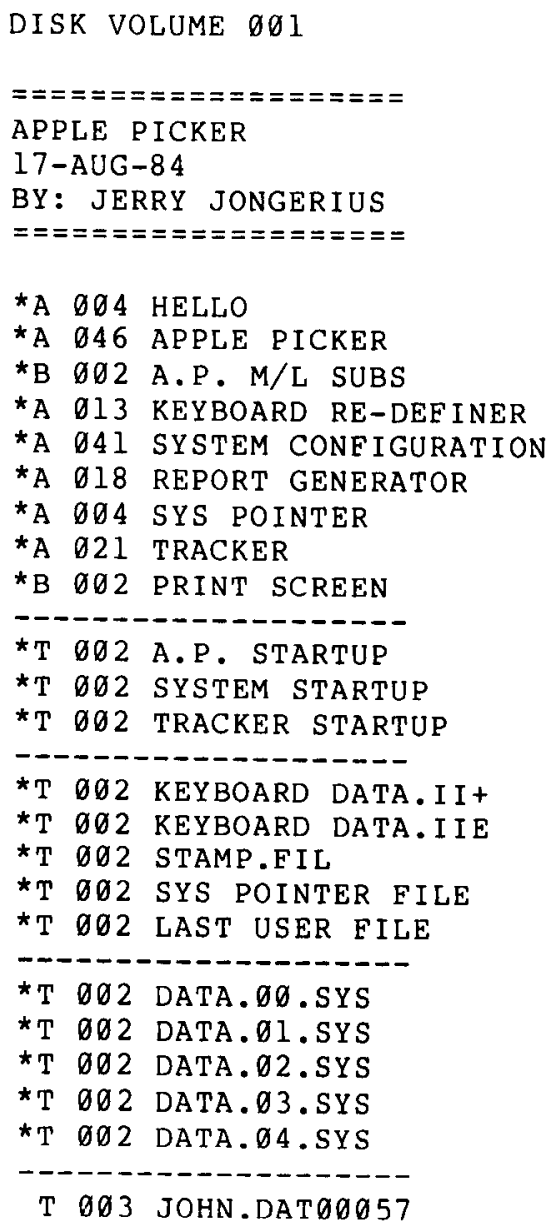

Figure 1. Catalog listing for all programs and support files on the Apple Picker diskette. The $* * n$ indicates that a file is locked. Programs preceded by " $A$ ” are written in Applesoft BASIC. Programs preceded by "B" are binary image files. All listings preceded by "T" are text files. 


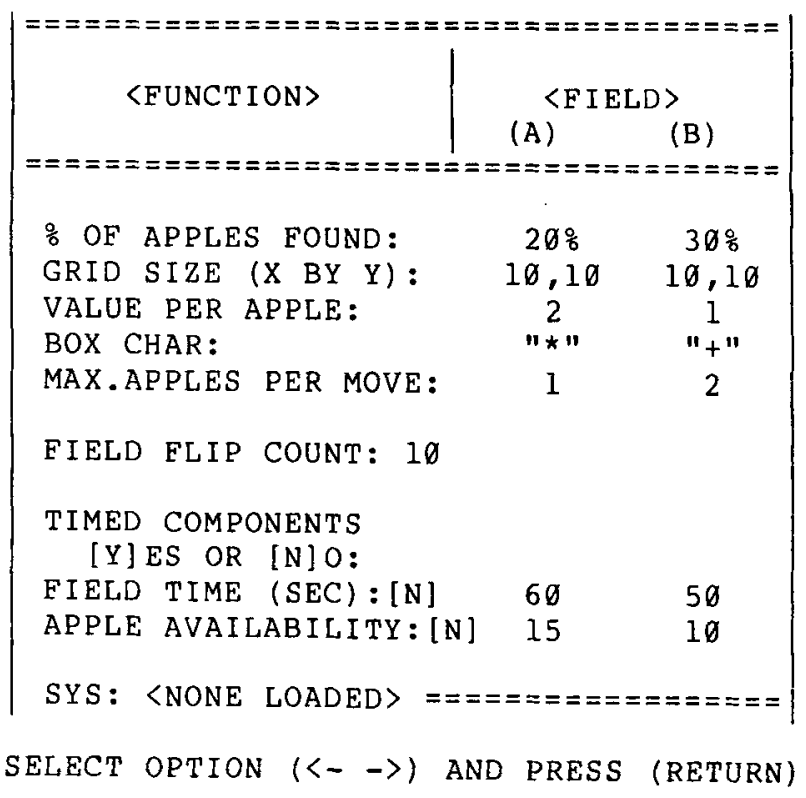

Figure 2. Sample System Data File. Percentage of apples found is used to set random ratio requirements. Grid size defines orchard size. Value per apple determines how many apples are found when a picking occurs. Box character represents the screen character for each orchard. Maximum apples per move defines how many apples may be picked from one tree before moving away. Field flip count determines the number of orchard $A$ or $B$ components during a session. Timed components allows one to specify ratio or interval schedules. Field time specifies the duration of components on multiple schedules. Apple availability specifies the random interval requirement. ${ }^{3}$

Five such files have been created and are stored at a different location on the diskette. Using this program, one may load an already existing System Data File into the program for modification, modify the old version of a file, and save the new version. The left and right arrow keys, along with the return key, are used to select one of these functions. In modifying a file, these same keys are used to select a specific parameter for modification.

REPORT GENERATOR. This program produces a report of the subject's responding during the session. A copy of the System Data File that was used is printed at the top of the page. Following this is a listing of the subject's responding in each orchard throughout the entire session. That listing includes a record of the following: time spent in the field; total moves, including the number of up, down, left, and right moves; the number of apples picked; the number of attempted pickings; and the number of worms encountered. Finally, a summary of responding in orchards $A$ and $B$, across all individual orchard presentations, is printed.

SYS POINTER. This short program allows the experimenter to set up a pointer to a specific System Data File. When Apple Picker is run, it automatically uses the System Data File currently loaded into the SYS POINTER program.

TRACKER. Using this program, one may generate a report that shows where the subject has moved in orchards A and B during the session. First, the System Data File is printed; then, in graphic form, a map of both orchards is printed along with tracings of the actual moves within each orchard. Finally, a verbal description of up, down, left, and right moves during each orchard presentation is printed.

In addition to these seven BASIC programs, two 6502 machine language programs are listed in this section of the catalog. A.P. M/L SUBS is a routine that allows Apple Picker to use two text pages and includes a sound routine. PRINT SCREEN is a routine used by TRACKER to print the two text pages side by side.

\section{EXEC Files}

This section of the catalog contains three EXEC start-up procedures necessary to start up Apple Picker (A.P. STARTUP), System Configuration (SYSTEM STARTUP) and Tracker (TRACKER STARTUP). These command procedures are executed from the HELLO program.

\section{Apple Picker Accessory Files}

The data needed by Apple Picker and its supporting programs are contained in this section. A description of each file follows:

Keyboard Data II + . This file contains six singlecharacter records that represent the keyboard control data for the Apple II+, as follows:

\begin{tabular}{|c|c|c|}
\hline Record number & Normal value & Function \\
\hline 1 & I & to move the cursor up \\
\hline 2 & $\mathbf{M}$ & to move the cursor down \\
\hline 3 & $\mathbf{J}$ & to move the cursor left \\
\hline 4 & K & to move the cursor right \\
\hline 5 & (space) & to pick an Apple \\
\hline 6 & (ESC) & to switch orchards \\
\hline
\end{tabular}

Keyboard Data IIe. This is the same as above, except for the Apple IIe.

Stamp.fil. This file contains one record, a number from 1 to 99999 , which is used to create a unique file name for each subject's test session (i.e., "JOHN.DAT00001"). Each time Apple Picker is run, this number is read, and then the file is updated to the old number plus one.

Sys Pointer File. This one-record file contains the file name of one of the System Data Files. One can create and store several System Data Files and then use this file to point to the one system file that Apple Picker will use during the session.

Last User File. This one-record file contains the User Data File (described below) for the previous subject. In generating a report, one is asked to type in the name of a User Data File. The full name may be typed in, or one may press "return" to generate a report of the last subject's data. The report-generating programs will auto- 
matically look to this file and accept the name just as though it had been typed in.

\section{System Data Files}

The files in this section of the catalog contain the system data needed in order for Apple Picker to work properly. As described above, Sys Pointer File points to one of these files. The five files in this section have been labeled DATA.00.SYS through DATA.04.SYS. It is suggested that one should modify these five files using SYSTEM CONFIGURATION rather than create new System Data Files for each experimental paradigm.

\section{User Data Files}

This section contains all of the User Data Files. Each time a subject uses Apple Picker, a file that records all responding is created as described in REPORT GENERATOR. Because a new file is created every time Apple Picker is run, it may be necessary to periodically DELETE some of the older User Data Files to allow room for new ones. If needed, these older User Data Files may be transferred to another diskette for later recall.

\section{PROGRAM AVAILABILITY}

The programs and supporting files described here may be obtained by sending an Apple-initialized diskette to the first author. It is our hope that others will begin testing and modifying Apple Picker to suit their various research interests.

\section{REFERENCES}

HeRRnSTEIn, R. J. (1970). On the law of effect. Journal of the Experimental Analvsis of Behavior, 13, 243-266.

MCSWEENEY, F. K. (1978). Negative behavioral contrast on multiple treadle-press schedules. Joumal of the Experimental Analysis of Behavior, 29, 463.473.

Norman, W. D., \& MCSweeney, F. K. (1978). Matching, contrast, and equalizing in the concurrent lever-press responding of rats. Journal of the Experimental Analysis of Behavior, 29, 453-462.

\section{NOTES}

1. The probability of finding a worm, given that an apple was not picked, may be changed by modifying line 5152 of Apple Picker. To set the probability to 0.0 modify this line as follows:

$$
\text { "IF(-.1 < RND (1)) THEN } 5160 "
$$

For all probabilities $>0.0$, simply insert that number into the statement in place of -.1 (e.g., for $p=.2$, insert .2).

2. If a joystick is not available, Apple Picker must be modified as follows to remove the joystick handling routine:

(1) Delete lines 50920-50929 and line 52010

(2) Add line 50920: "IF PEEK (-16384) > 127 THEN 50990"

3 . If a concurrent schedule is required, respond with " $N$ " to the Timed Components parameter. The ESC key is then used during APPLE PICKER to switch between orchards. A response of " $Y$ " to Timed Components institutes a multiple schedule. 